\title{
RENOVACIÓN TIPOLÓGICA Y FUNCIONAL EN CASCOS HISTÓRICOS: PARTICIPACIÓN, CONFLICTOS Y RE- SULTADOS. UN ANÁLISIS A TRAVÉS DE ESTUDIOS DE CASO EN LAS CIUDADES DE CÁCERES Y BADAJOZ
}

\author{
Diego A. BARRADO TIMÓN \\ Departamento de Geografía. Universidad Autónoma de Madrid \\ diego.barrado@uam.es
}

\author{
Kenia GARCÍA BALTODANO \\ Departamento de Geografía. Universidad Autónoma de Madrid \\ kenia.garcia@estudiante.uam.es \\ David PORRAS ALFARO \\ Departamento de Geografía. Universidad Autónoma de Madrid \\ david.porras@uam.es
}

Recibido: $14 / 10 / 2013$
Aceptado: $08 / 01 / 2014$

RESUMEN: El presente texto estudia los procesos de integración y aceptación social de nuevas funciones y lenguajes arquitectónicos en conjuntos históricos con un alto grado de protección, con una imagen y un simbolismo muy consolidados en relación con el espacio urbano y con gran significado para la mayor parte de la población. A partir de los estudios de caso del hotel Atrio Relais \& Châteaux de Cáceres y de la Facultad de Biblioteconomía y Documentación de Badajoz, se profundiza en las posturas de los agentes sobre dichos proyectos, así como en los procesos de conflicto y negociación derivados. Con base en este análisis empírico se sistematizan las posiciones a favor y en contra, y se plantean conclusiones referidas al papel que dicha participación social ha tenido tanto en la configuración final de cada uno de los proyectos como en cuanto a la aceptación social de los mismos. El texto se cierra con una valoración de los casos analizados desde el punto de vista social y ambiental, y con una serie de conclusiones y elementos para posteriores investigaciones y debates. PALABRAS CLAVE: Conjuntos históricos, intervención, funcionalidad, simbolismo, Badajoz, Cáceres

1 El presente texto se incardina en el proyecto de investigación Las ciudades españolas en la etapa autonómica (1978-2012). Dinámicas, procesos y políticas (URBSPAIN), financiado por el Plan Nacional de Investigación I+D+i del Ministerio de Economía y Competitividad para el período 2010-2013 (Código CSO 2009-11261-Subpr. GEOG). 
TYPOLOGICAL AND FUNCTIONAL RENEWAL INHISTORIC CENTERS:

PARTICIPATION CONFLICTS AND RESULTS. AN ANALYSIS THROUGH CASE STUDIES IN THE CITIES OF CÁCERES AND BADAJOZ

ABSTRACT: This paper is devoted to research the processes of integration and social acceptance of the new functions and the latest architectural languages in those historical sites with a high degree of protection, with a very consolidated image and symbolism amongst the majority of its population in relation to the urban space as a whole. From the case studies of Caceres Atrio Relais \& Châteaux Hotel and the University of Extremadura Facultad de Biblioteconomía y Documentación in Badajoz, this paper goes into detail about the different strategies that both for and against agents follow, as well as about the processes of conflict and negotiation these originate. The positions in favor or against where systematized based on the empirical analysis. The conclusions are based on the role that social participation has had on the final configuration and social acceptance of the projects. The text is closed with social and environmental evaluations of the different projects analyzed across it, and a range of conclusions and elements for further research and discussion.

KEY WORDS: Historical sites, intervention, function, symbolism, Badajoz, Caceres.

\section{INTRODUCCIÓN: OBJETIVOS E HIPÓTESIS DE LA INVESTI- GACIÓN}

Este texto analiza dos proyectos de intervención arquitectónica y urbanística en espacios monumentales y conjuntos históricos extremeños: la construcción del hotel Atrio Relais \& Châteaux en la plaza de San Mateo, un alojamiento de cinco estrellas y restaurante en la ciudad amurallada de Cáceres; y la Facultad de Biblioteconomía y Documentación de la Universidad de Extremadura, que junto con la Biblioteca Regional ocupó y modificó un hospital militar decimonónico situado dentro de la Alcazaba almohade de Badajoz. En ambos casos, como se verá de forma más detallada en páginas posteriores, se trata de espacios con un alto nivel de protección, ya que la primera ciudad fue inscrita en la Lista del Patrimonio Mundial en 1986, y el conjunto monumental y arqueológico pacense es Bien de Interés Cultural (BIC) desde 1931.

Los objetivos científicos en los que se inscriben los estudios de caso señalados se centran en dos aspectos fundamentales: por un lado, la valoración de las políticas de intervención en cascos históricos y en conjuntos monumentales, haciendo especial hincapié en las dificultades existentes para integrar nuevas funciones y lenguajes arquitectónicos en espacios muy institucionalizados y con una imagen y un simbolismo consolidado. En segundo lugar, directamente derivado de lo anterior, la conflictividad y los procesos de participación social desarrollados, con el fin de valorar su posible trascendencia en la configuración final del proyecto, además de su mejor o peor aceptación e integración funcional, económica, urbanística y simbólica en el conjunto urbano. 
En cuanto al primero de los aspectos señalados, la intervención en espacios urbanos patrimoniales, es una competencia asumida de forma exclusiva por las comunidades autónomas, como refleja para los casos analizados la Ley de Patrimonio Histórico y Cultural de Extremadura ${ }^{2}$ en su exposición de motivos. Sin embargo, dicha exclusividad se ve notablemente atenuada por competencias concurrentes tanto del Estado central, derivadas de la propia Constitución y de la Ley del Patrimonio Histórico Español ${ }^{3}$, como municipales, que en el caso expreso de los conjuntos históricos se concretan en aspectos tan relevantes como la redacción y aprobación inicial de los planes de urbanismo o especiales y en la concesión de licencias.

Del análisis empírico realizado no se pudo constatar la existencia de una política explícitamente definida por parte de la Comunidad Autónoma de Extremadura en materia de conservación y protección del patrimonio inmueble y urbano, y de forma mucho más puntual sobre los conjuntos históricos. A falta de una aproximación amplia que permitiese concretarla de forma implícita a partir de las intervenciones desarrolladas, cabe apuntar que el modelo legalmente definido para la intervención obliga a los correspondientes ayuntamientos a la redacción de un plan especial de protección ${ }^{4}$ del área afectada, cuya aprobación definitiva requerirá el informe favorable de la Consejería de Cultura y Patrimonio. Al margen de las exigencias habituales propias de los planes especiales para conjuntos históricos, como el mantenimiento del ambiente y el paisaje sin admitir de forma general modificaciones en las alineaciones, edificabilidad o parcelario, el artículo 41 de la Ley 2/1999 establece algunos aspectos sustanciales para el análisis que aquí se pretende abordar, como son la necesidad de priorizar los usos públicos para la instalación en los edificios y espacios que fuesen aptos para ello, así como contemplar las áreas de rehabilitación integrada que permitiesen recuperar la función residencial y las actividades económicas.

El segundo de los objetivos fundamentales de la investigación es, como se dijo más arriba, analizar la intervención de los actores individuales, colectivos e institucionales, valorando sus estrategias y el papel que desempeñaron tanto en el proceso como en la final aceptación de los resultados. Tal y como se irá viendo,

2 Ley 2/1999, de 29 marzo, de Patrimonio Histórico y Cultural de Extremadura, parcialmente modificada por la Ley 3/2011, de 17 de febrero, de modificación parcial de la Ley 2/1999, de 29 marzo, de Patrimonio Histórico y Cultural de Extremadura.

3 Ley 13/1985, de 25 de junio, del Patrimonio Histórico Español.

4 Dicho plan especial de protección puede sustituirse por la redacción de un documento urbanístico general, siempre y cuando en el ámbito delimitado cumpla con las exigencias que la Ley establece para el plan especial. 
ninguno de los proyectos con los que se trabaja surgió de iniciativas de participación que pudiesen catalogarse de bottom-up, o derivadas de un modelo consensuado de intervención general sobre la ciudad histórica y sobre su integración en el conjunto urbano. Antes al contrario, podrían ser catalogadas de actuaciones no solo puntuales sino oportunistas ${ }^{5}$, en la medida en que surgen en un contexto en el que confluyen de forma favorable tanto los actores como una situación política, administrativa y financiera propicia. Sin embargo, lo cierto es que finalmente se produjo un complejo proceso de participación social entendida en un sentido muy amplio, que fue desde la crítica pasiva y activa al proyecto y sus resultados (folletos, manifestaciones, intervención en los medios, denuncias judiciales) hasta el trabajo de algunos agentes en el rediseño y la definición final de los mismos.

\section{MARCO TEÓRICO PARA LA DISCUSIÓN E HIPÓTESIS DESA- RROLLADA}

Como señala J. L. De Las Rivas (2009), la convivencia de los centros históricos con la contemporaneidad obliga a plantearse la idea de "ciudad sobre ciudad", y con ella, los importantes desafíos en torno a los conflictos derivados de la superposición de funciones, usos y estructuras provenientes de la combinación de las estrategias de conservación con las de puesta en valor de los elementos que configuran el patrimonio urbano. De hecho, esta problemática ha sido objeto de un reciente proyecto impulsado por el Grupo de Ciudades Patrimonio de la Humanidad de España y el Ministerio de Cultura, del que se ha derivado una carta programática sobre la integración de la arquitectura contemporánea en las ciudades Patrimonio de la Humanidad, en donde se plantean, entre otros aspectos, la necesidad de ofrecer respuestas contemporáneas a los problemas arquitectónicos y socioeconómicos de las ciudades históricas, así como la de abordar los proyectos emblemáticos de un modo transparente que dé entrada a la participación social ${ }^{6}$.

De manera general, puede señalarse la existencia de dos grandes planteamientos en torno a esta cuestión. El primero, calificado de modelo defensivo o de

5 Dicho calificativo no debe ser entendido en su habitual vertiente negativa, en el sentido de prescindir de principios básicos y fundamentales.

6 El referido proyecto, que lleva por título "Estrategias para la incorporación de la arquitectura contemporánea en las ciudades Patrimonio de la Humanidad de España" (2009) fue dirigido por los arquitectos Francisco Pol y Asunción Rodríguez. Parte de sus conclusiones están recogidas en la "Carta para la integración de la arquitectura contemporánea en las ciudades Patrimonio de la Humanidad" http://www.femp.es/files/566-990-archivo/Arq_contemporanea_ciudades_patrimonio.pdf. 
conservación pasiva, reafirma el tratamiento de la ciudad histórica desde la lógica de la excepcionalidad y la monumentalidad, privilegiando la conservación y concentrándose fundamentalmente en los aspectos culturales y patrimoniales. Frente a esta postura se sitúa la en ocasiones denominada nueva cultura de la ciudad, que reconoce los valores de la ciudad histórica pero da prioridad a la integración social y funcional de esta pieza en el conjunto urbano ${ }^{7}$, cuestionado incluso su centralidad desde el punto de vista funcional (CASTRILlo y JiMÉNEZ, 2011). De la aceptación de una u otra perspectiva, que evidentemente nunca es absoluta, se derivan posiciones ideológicas de gran significación para esta investigación, concretadas en respuestas diferenciadas a cuestiones tan importantes como qué funciones y qué lenguajes arquitectónicos serían admisibles en los conjuntos históricos; así como la muy diferente valoración de una tipología de intervenciones concretas y puntales que, a medio camino entre la arquitectura y el marketing urbano, buscan sobre todo potenciar el simbolismo y la iconicidad de una nueva monumentalidad contemporánea.

Como señalan M. A. Troitiño y J. García (1998) los centros históricos, además de sus valores culturales excepcionales, presentan características sociales que deberían ser consideradas en conjunto. Entre estas pueden citarse su carácter simbólico, vinculado a la representatividad y significado para la población, reflejo de la realidad social; su carácter vivencial, al ser espacios intensamente vividos tanto por residentes como por visitantes; su multifuncionalidad, debida a la variedad de actividades que coexisten y que aportan riqueza a la vida urbana; y finalmente su heterogeneidad social, debida a la propia diversidad interna y a las transformaciones de diferente naturaleza llevadas a cabo a través del tiempo. Todos estos factores llevan a considerar que las actuaciones dentro de los espacios heredados requieren de propuestas basadas en estudios minuciosos del entorno, que permitiesen dar respuestas económica, funcional y estéticamente viables, y que a su vez puedan ser simbólicamente aceptadas por el conjunto de la población.

Sin embargo, las tendencias actuales parecen bascular la intervención sobre los centros históricos hacia un modelo de conservación pasiva (CASTRILLO Y

7 Vale la pena señalar la posición de diversas Cartas Internacionales que manifiestan la necesidad de no desligar los centros históricos de su contexto urbano, paisajístico y territorial, así como el papel de los diversos actores que intervienen en estos sitios. Al respecto pueden consultarse la Carta de Cracovia o "Principios para la Conservación del Patrimonio Construido" (2001), el Memorando de Viena sobre "El Patrimonio Mundial y la arquitectura contemporánea. Gestión del paisaje histórico urbano" (2005), las "Recomendaciones sobre Paisaje Urbano Histórico", presentadas en la Actas de la $36^{\circ}$ Reunión de la Conferencia General de la UNESCO (2011) y los "Principios de La Valeta para la salvaguarda y gestión de las poblaciones y áreas urbanas históricas” (2011). 
JiMÉnEZ, 2011), con la renuncia a una idea de planeamiento global que es sustituido por el recurso a actuaciones individualizadas pero estratégicamente localizadas en el espacio, en suma, a la suplantación del planteamiento urbanístico por el proyecto arquitectónico (Álvarez Mora, 2000). Se trata, en realidad, de intervenciones extraordinarias generalmente dirigidas a acoger funciones públicas o servicios de alto nivel, buscando la nueva monumentalidad y el simbolismo asociados a los lenguajes arquitectónicos contemporáneos, así como con una cierta propensión hacia la elitización de algunos ámbitos de la ciudad monumental.

Estos procesos brevemente descritos se van a analizar en los dos proyectos presentados, centrando la atención en la valoración social suscitada y en los procesos de negociación y conflicto. Para ello, se ha construido un marco metodológico que presta atención a dicha valoración social en dos aspectos fundamentales: en primer lugar desde el punto de vista de la función, en relación con su posible papel en cuanto a la conservación y revitalización de la ciudad histórica; posteriormente, a la integración arquitectónica y urbanística del edificio, es decir, a su capacidad para adaptarse o superponerse a la trama heredada.

La primera de las líneas de análisis referidas se ha denominado ideológica, y se centra esencialmente en las diferentes visiones de los agentes sobre cómo deben ser las formas de intervención en los centros históricos. Estas posturas se sistematizan en la oposición de intervencionismo frente a conservacionismo ${ }^{8}$, paralelas en cierta medida a las corrientes de pensamiento de nueva cultura de la ciudad y monumentalismo ya comentadas. Los elementos sobre los que se concretará este análisis son, de manera fundamental, la aceptación o no de nuevas funciones en los centros históricos, y la valoración positiva o negativa de la imagen y la monumentalidad de la nueva arquitectura contemporánea, en contraposición con el simbolismo del patrimonio heredado.

El segundo elemento de sistematización se dirige a los aspectos técnicos. De forma genérica se pretende valorar las posturas de los agentes en relación con la concreción de sus respectivas posiciones ideológicas, que de manera fundamental hacen referencia a las perspectivas legales, urbanísticas y arquitectónicas de las intervenciones desarrolladas. Se concreta en situaciones como el apoyo o rechazo a los cambios puntuales en el planeamiento para incluir nuevas funciones, o la aceptación de volúmenes, formas, huecos y materiales novedosos en los espacios monumentales.

8 Tanto intervencionismo como conservacionismo se usan aquí simplemente a modo de categorías neutras, entendidas como pares de opuestos sin ninguna intención valorativa, y por supuesto, sin ninguna de las connotaciones negativas que a cualquiera de las dos posturas se les suelen atribuir desde posiciones contrarias. 
Podría concluirse señalando que desde el punto de vista ideológico se pretende valorar la posición de cada agente ante lo que se quiere hacer, el proyecto y las motivaciones que lo mueven; mientras que la vertiente técnica valora su opinión sobre lo que se ha hecho y cómo se ha hecho. Evidentemente, ambas perspectivas están directamente conectadas, en el sentido de que no se acepta la posibilidad teórica de una solución técnica supuestamente aséptica y al margen de las posiciones ideológicas de partida. El cuadro de las diferentes combinaciones posibles, a favor y en contra, quedaría del siguiente modo (Cuadro 1).

Cuadro 1. Posibles combinaciones de las posiciones encontradas en los proyectos analizados.

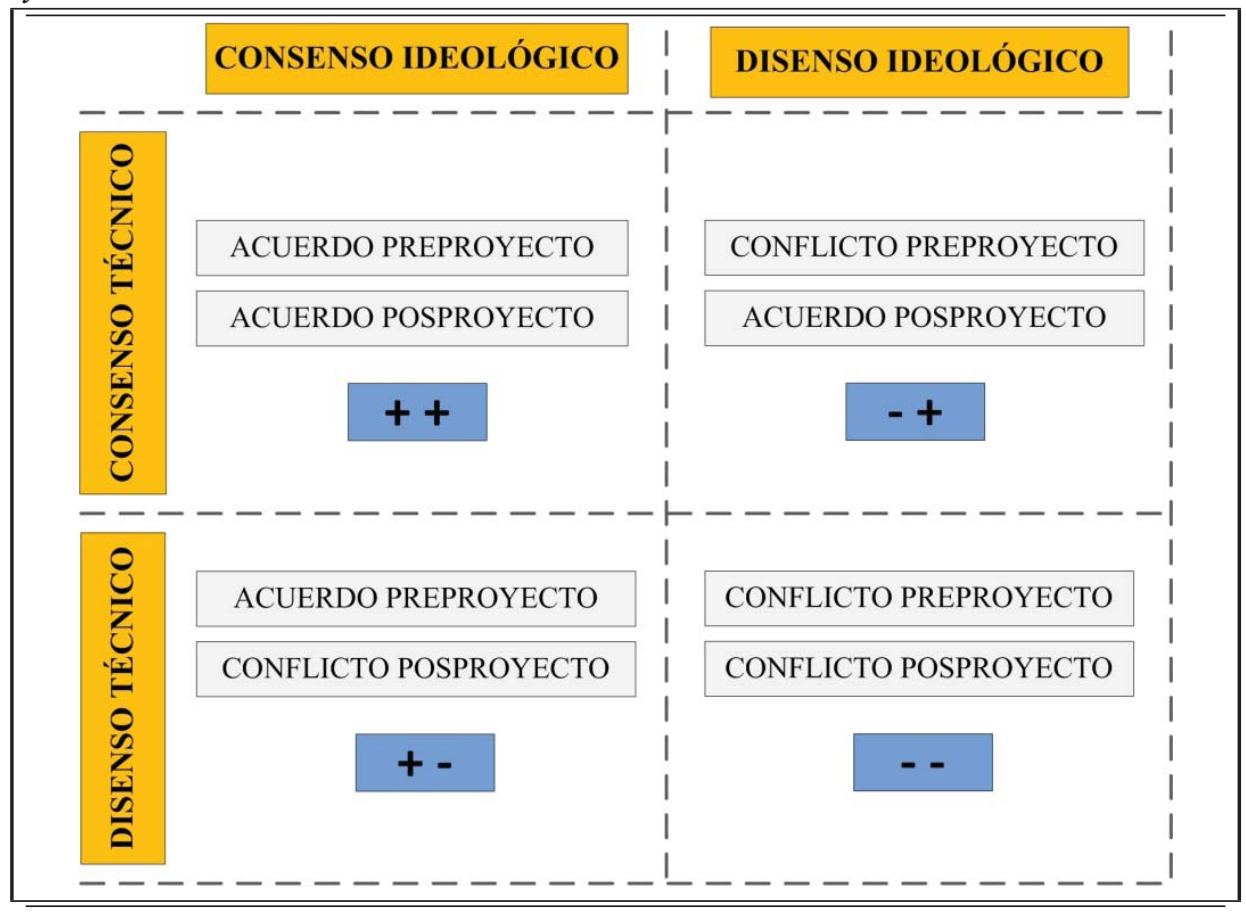

FUENTE: Elaboración propia.

De acuerdo con estos planteamientos la hipótesis que se propone es la siguiente: la aceptación consensuada y favorable de proyectos que introduzcan nuevas funciones y lenguajes arquitectónicos en conjuntos históricos, así como la admisión social de un nuevo simbolismo derivado de la monumentalidad contemporánea, depende de la combinación de posiciones ideológicas y técnicas, junto con el papel que los diferentes actores hayan representado en la definición final del proyecto. 
Se asume que la discusión, reevaluación y modificación, atendiendo a diferentes perspectivas y sensibilidades, de un proyecto arquitectónico y urbanístico que vaya a modificar la imagen y funcionalidad de espacios tan emblemáticos como los cascos históricos, solo puede ser positiva. Incluso en el caso de que el resultado final no satisfaga a todos los actores, y algunos sigan manteniendo una oposición al mismo más o menos manifiesta y pública, la realidad es que la readaptación técnica del proyecto, a modo de encaje de piezas sobre diferentes aspectos (legal, urbanístico, arquitectónico, estético, volumétrico, etc.), suele conllevar su integración simbólica en la imagen que el centro histórico proyecta sobre el conjunto de la ciudad. Por el contrario, cuando de la discusión sobre los aspectos ideológicos no se deriva una nueva forma de encaje técnico del proyecto, este presenta más dificultades para integrarse simbólicamente en la imagen urbana, sean cuales sean sus resultados funcionales.

\section{METODOLOGÍA Y FUENTES}

La valoración social de las intervenciones elegidas ha sido objeto de un análisis cualitativo, desarrollado a través de entrevistas con actores que, tras una primera aproximación a los casos, se consideraron clave. No obstante, la disposición a la participación ha sido desigual, dado que se trata de procesos muy conflictivos y que, con grados diferentes como se verá más adelante, aún siguen abiertos. Positivamente, puede señalarse la apertura de los agentes sociales, tanto técnicos como asociaciones, mientras que las reticencias han sido mayores con empresas e instituciones; apuntar, en este sentido, dos casos importantes y que evidentemente afectaron al proceso de investigación. El hotel Atrio Relais \& Châteaux de Cáceres, tras varias conversaciones con la directora del mismo en las que mostró una clara disposición a la participación, remitió a uno de los propietarios que indicó que prefería no volver sobre estos temas; sin embargo, tras dicho rechazo inicial mantuvo una conversación telefónica cercana a la media hora en la cual se cubrieron parte de los contenidos necesarios para la investigación. El segundo actor que planteó problemas fue el Ayuntamiento de Badajoz, pues tras varios contactos telefónicos y por correo electrónico con su portavoz, que remitió al concejal de Urbanismo, dejó de contestar a los mismos y finalmente no pudo ser entrevistado.

El cuadro final de entrevistas desarrolladas es el siguiente:

- Facultad de Biblioteconomía y Documentación de la Universidad de Extremadura. 
- Antonio Manzano (en Badajoz, el 12 de junio de 2013), miembro de la Asociación Amigos de Badajoz.

- Manuel Sosa (en Badajoz, el 13 de junio de 2013), concejal de Izquierda Unida (IU) en el Ayuntamiento de Badajoz.

- Agustín Vivas (en Badajoz, el 14 de junio de 2013), decano de la Facultad de Biblioteconomía y Documentación.

- Javier Teijeiro (conversación telefónica, el 21 de junio de 2013), arquitecto y perito de la Asociación Amigos de Badajoz.

- Hotel Atrio Relais \& Châteaux de Cáceres.

- Antonio Campesino (en Cáceres, el 29 de mayo de 2013), catedrático de la Universidad de Extremadura, y en el momento de desarrollo del proyecto miembro de la Comisión Municipal de Seguimiento del Plan Especial y del Comité Español del Consejo Internacional de Monumentos y Sitios (ICOMOS).

- Juan Antonio 'Toño' Pérez (conversación telefónica, el 10 de junio de 2013), propietario y chef del hotel Atrio Relais \& Châteaux.

- Antonio Díaz y otros miembros de la Asociación para la Defensa de la Naturaleza y los Recursos de Extremadura (ADENEX) (en Cáceres, el 13 de junio de 2013).

El proceso de investigación sobre los actores y sus posiciones, así como sobre el desarrollo de las polémicas suscitadas, se ha completado con fuentes provenientes de una abundante documentación periodística de ámbito regional y nacional, que ha suministrado además importante material gráfico. El tercer gran grupo de fuentes proviene de informes técnicos de carácter urbanístico, arquitectónico o patrimonial, así como varias sentencias judiciales relativas al caso de Badajoz.

Finalmente, se realizó un intenso trabajo de campo en ambas ciudades, con el fin de valorar los diferentes aspectos de los proyectos sobre los que se apoya el análisis social y ambiental, que se incluye en la parte final del artículo.

\section{LA FACULTAD DE BIBLIOTECONOMÍA DE BADAJOZ Y EL HOTELATRIO RELAIS \& CHÂTEAUX DE CÁCERES: PROYEC- TOS, AGENTES, ACUERDOS Y CONFLICTOS}

En los últimos años se han producido algunas intervenciones arquitectónicas y urbanísticas en varios de los conjuntos monumentales extremeños que han generado una cierta repercusión social y mediática, dando como resultado formas de participación que pueden ser vistas como muestra del interés que para la so- 
ciedad ha cobrado el patrimonio urbano, su conservación y su puesta en valor. De entre ellas se ha optado por escoger como estudios de caso una en Badajoz y otra en Cáceres, en la medida en que ambas tuvieron y tienen un largo recorrido, dejando tras de sí una extensa documentación y muy diferentes posibilidades de análisis, que han permitido plantearse las cuestiones teóricas y metodológicas apuntadas en las páginas precedentes.

\section{IV.1 La Facultad de Biblioteconomía en la Alcazaba de Badajoz: el problema de la integración de una nueva función en un conjunto arqueológico}

La Alcazaba, origen de la ciudad y BIC desde 1931, es una ciudadela almohade de ocho hectáreas construida en el siglo XII sobre el cerro de la Muela. Derivado de la función defensiva que ha marcado históricamente a Badajoz, domina tanto el río Guadiana como el casco, un conjunto de calles paralelas y perpendiculares a las curvas de nivel que descienden en forma de abanico desde la Plaza Alta, adosada al flanco sur de la Alcazaba, hasta los límites marcados por la muralla y los baluartes Vauban (PAGÉs y CAMPESINO, 1990).

Se trata de un conjunto urbano en el que se han detectado altos niveles de vulnerabilidad en todos los análisis realizados, y que especialmente en su parte más elevada presenta evidentes problemas sociales, residenciales y funcionales, así como una imagen muy deteriorada que se fue haciendo más patente a medida que perdía funciones urbanas, como el mercado situado en la Plaza Alta (HerNÁNDEZ AJA et al., 2011), y que llevó a gran parte de los habitantes de la ciudad a evitar totalmente dicha zona hasta hace relativamente poco tiempo. La lista de intentos para atraer nueva población y funciones, favoreciendo la rehabilitación del barrio, es muy larga, la mayoría con muy escaso éxito a la hora de reintegrarlo en el conjunto de las dinámicas urbanas. ${ }^{9}$. A modo de ejemplo pueden citarse un plan especial redactado en 1990 y nunca aprobado (PAGÉs y CAMPESINO, 1990); el actual Plan Especial, integrado en el Plan General Municipal de 2007; diversos proyectos de rehabilitación desde 1979, en donde destaca el de las viviendas de la Plaza Alta ${ }^{10}$; un plan URBAN aprobado en 1995; o la localización en el recinto de la Alcazaba del Museo Arqueológico Provincial de Badajoz.

9 Puede ampliarse la información sobre la situación del área y las estrategias de recuperación en la información sobre Análisis urbanístico de barrios vulnerables realizado por el Departamento de Urbanística de la Universidad Politécnica de Madrid http://habitat.aq.upm.es/bbvv/ bbvv_112.html.

${ }^{10}$ Puede consultarse el complejo proceso de rehabilitación arquitectónica y urbanística de la Plaza Alta y sus alrededores en J. M. González González (2006). Señalar, en este sentido, otro proyecto recientemente finalizado e igualmente polémico desde el punto de vista arquitectónico y 
Es en este contexto en el que hay que entender la idea, expresada por primera vez en 1995, de localizar en la Alcazaba la recién creada Facultad de Biblioteconomía de la Universidad de Extremadura ${ }^{11}$, que en ese momento no tenía una sede en el campus situado al oeste de la ciudad. Se pretendía que el tránsito de estudiantes y profesores a través del casco modificase el paisaje social de la parte alta, favoreciendo poco a poco su recuperación residencial y económica mediante pisos para estudiantes y pequeño comercio especializado; una idea que fue catalogada en diversos ámbitos de "descabellada pues para buena parte de la ciudadanía subir a la Alcazaba daba miedo” (LópEz-LAGo, 2009).

Dicha idea $^{12}$ fue recogida por el gobierno autonómico, el alcalde de la ciudad y el rector de la Universidad de Extremadura, lo que implicaba un importante consenso institucional que incluía a los dos partidos de signo contrario que en ese momento se encontraban al frente del poder autonómico (PSOE) y local (PP). En este contexto de consenso político ${ }^{13}$ el Ayuntamiento compró el hospital militar decimonónico, un edificio protegido pero de relativamente escaso valor patrimonial $^{14}$ aunque situado en el interior de la fortaleza almohade, y lo cedió a la Junta. Esta cesión conllevó además la inclusión de la Consejería de Cultura como actor, y con ella la decisión de instalar junto con la Facultad de Biblioteconomía la Biblioteca Regional de Extremadura; dicha combinación de usos, perfectamente lógica desde un punto de vista funcional, puede estar en el origen de la necesidad de construir y levantar en altura nuevos cuerpos adosados al hospital para cubrir las funciones educativas y científicas, dado el alto porcentaje del antiguo edificio ocupado por la Biblioteca.

urbanístico, pero que ha tenido menos trascendencia social, como es la inserción en la muralla de la Alcazaba de una nueva estructura para la Concejalía de Cultura, en este caso accesible desde la Plaza Alta; lo que el arquitecto Javier Teijeiro calificó como el "otro cubo".

${ }^{11}$ En realidad, la primera vez que se realiza dicha propuesta fue en el arriba citado Plan Especial de Protección del Patrimonio Urbano de Badajoz (PAgÉs y CAMPEsIno, 1990), que entendía que los universitarios eran los únicos agentes capaces de revertir el proceso de deterioro y abandono del conjunto de la Alcazaba y la Plaza Alta. Como se señaló, dicho plan no fue aprobado.

${ }^{12}$ La historia del proceso de implantación de la Facultad, y del conflicto generado por la misma, está extraída en su mayor parte de LóPEz-LAGo, J. (2009). http://www.hoy.es/20091122/ badajoz/ajetreada-historia-cubo-20091122.html.

${ }^{13}$ El único grupo con representación municipal que ha presentado una posición crítica, no en lo ideológico - funcional pero sí en lo técnico, es IU.

${ }^{14}$ En un informe de la Real Academia de Bellas Artes de San Fernando, al que con posterioridad se hará referencia y contextualizará, el único elogio que parece encontrarse para él es el de "noble y sólido Hospital Militar". 
El proyecto arquitectónico, adjudicado tras un concurso al equipo formado por los arquitectos Enrique Colomés y Gonzalo Moure, planteaba derribar parte del hospital y levantar nuevas estructuras, algo a lo que ni el Ayuntamiento -que concedió la licencia-, ni la Comisión Provincial de Bienes Inmuebles y Patrimonio -que fue previamente consultada-, pusieron ningún obstáculo debido al escaso valor del mismo. No pareció pesar decisivamente en dicha decisión el hecho de que este se encontrase integrado en un conjunto arqueológico protegido como BIC desde 1931, que además presenta un enorme peso en la imagen y el simbolismo urbano dada su situación topográficamente elevada. En dicha comisión participó y votó a favor del proyecto el representante de la Asociación Amigos de Badajoz, que en el año 2000, y tras comprobar que la nueva estructura sobresalía por encima de la muralla y del conjunto de torres de la fortificación, pidió por primera vez la paralización de las obras. En la entrevista realizada con Antonio Manzano, reconocía las repercusiones de dicho voto a favor, pero aducía que de los planos y las maquetas presentadas no se podía deducir que el recrecimiento del edificio originario, y de forma muy especial que las zonas de nueva construcción, el popularmente conocido como 'Cubo' de Biblioteconomía, fuesen a modificar el perfil de la Alcazaba y de algunos de sus elementos más simbólicos, como la torre de Espantaperros ${ }^{15}$ (Imagen 1).

Imagen 1. Vista desde la Plaza Alta del cuerpo elevado del hospital y el 'Cubo' de nueva planta, y desde el interior de la Alcazaba en el que se aprecia su relación con la muralla y la torre de Espantaperros.
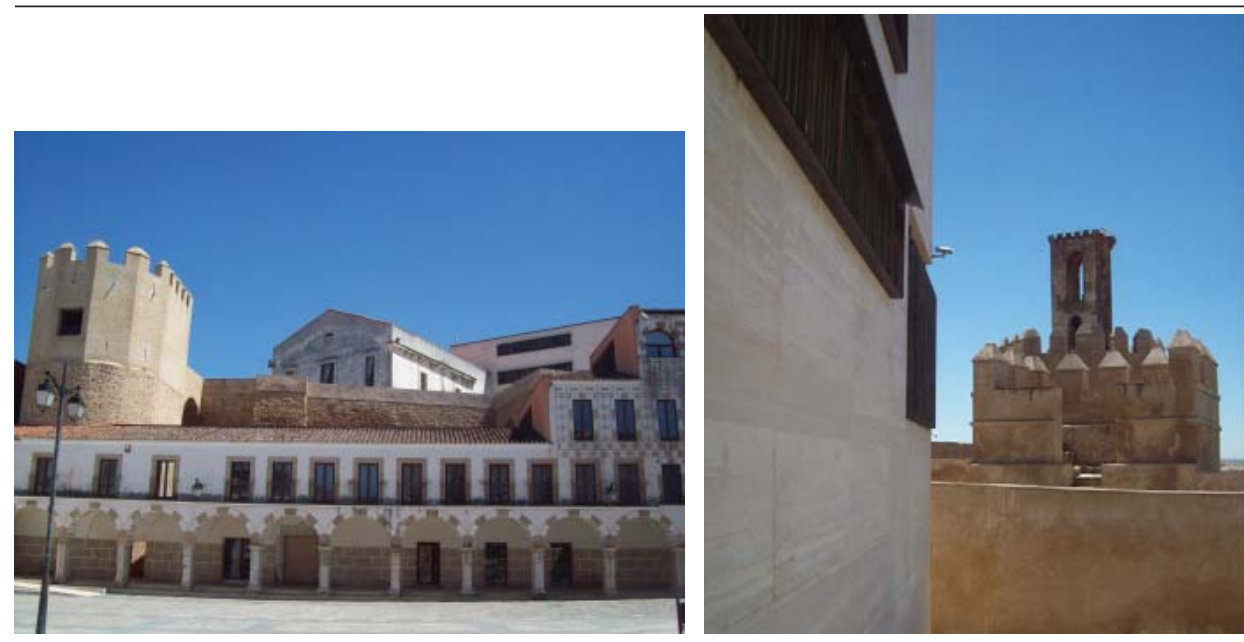

Fuente: Diego A. Barrado Timón.

15 La torre albarrana de Espantaperros es posiblemente el elemento arquitectónico más conocido y simbólico de la ciudad. 


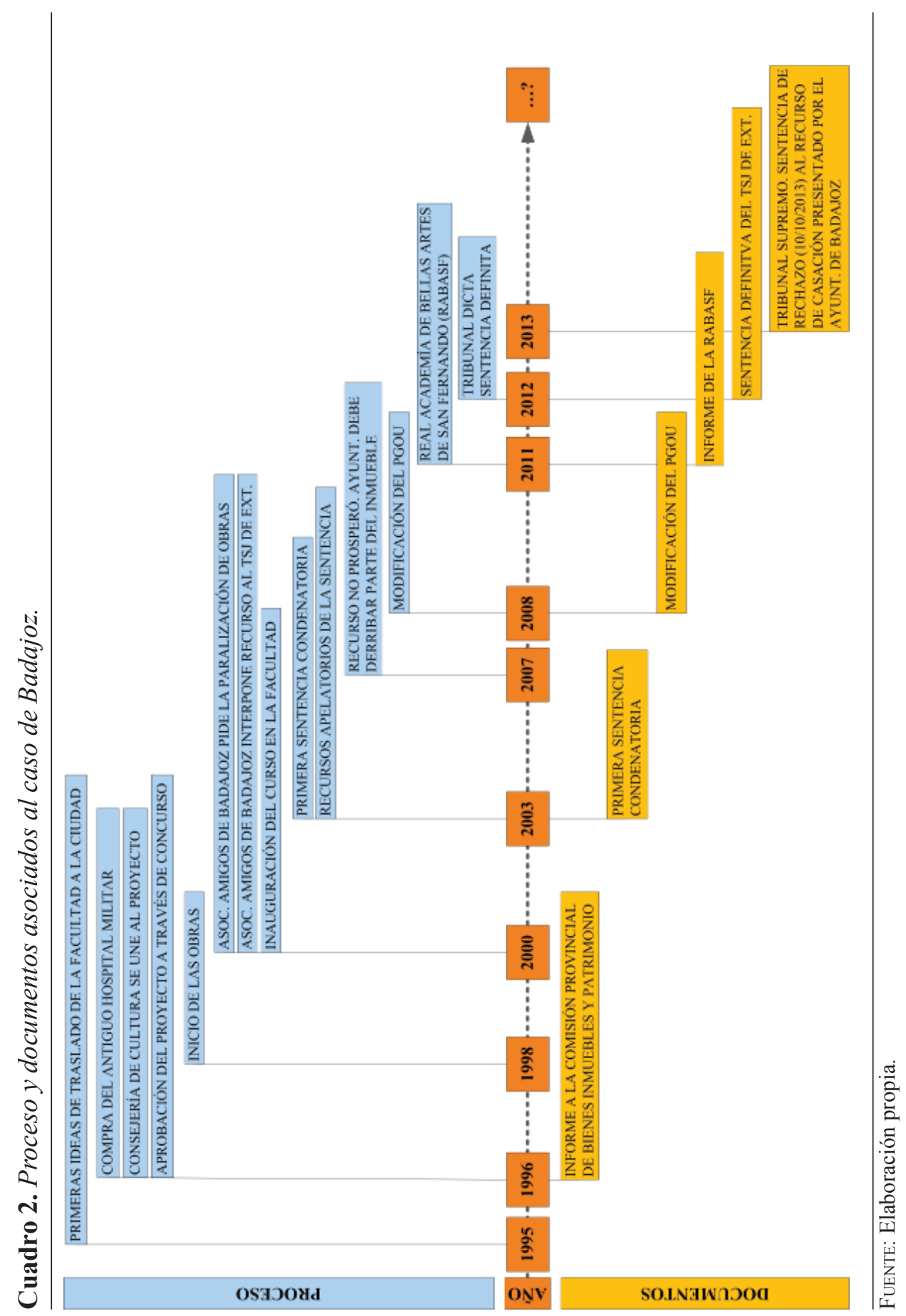

Polígonos. Revista de Geografia, 25 (2013); 57-89 
A partir de ese momento se inicia un tortuoso conflicto que llega hasta la actualidad (Cuadro 2), y que ha supuesto una intensa participación y una significativa presencia política, mediática y social del problema urbanístico, arquitectónico y paisajístico creado, incluyendo negociaciones más o menos secretas, manifestaciones, campañas, declaraciones y la creación de asociaciones y grupos a favor y en contra del proyecto.

Además, se produjo la judicialización del proceso en el año 2000, tan solo uno antes de la inauguración de la Facultad, cuando Amigos de Badajoz interpuso un recurso ante el Tribunal Superior de Justicia de Extremadura que finalizó con una condena a la corporación municipal que obligaba a restaurar el monumento a su estado anterior a las obras autorizadas; dicho fallo fue recurrido por el Ayuntamiento y la Junta de Extremadura, quienes finalmente obtuvieron una respuesta negativa que reafirmaba la necesidad de demoler parte del nuevo edificio, concretamente dos de las plantas que sobresalen por encima de la muralla (Imagen 2). En abril de 2008 el Tribunal Superior de Justicia de Extremadura dictó un nuevo auto que estimaba inejecutable el derribo, al no ser posible restaurar la situación inicial, asumiendo además el nuevo marco urbanístico que para la Alcazaba y sus elementos conllevaba el Plan General Municipal y Plan Especial de Protección del Casco Histórico aprobado en 2007. Este complejo recorrido judicial pareció llegar a su fin en octubre de 2009, cuando el Tribunal Supremo dictó un fallo inapelable que obligaba a la demolición, desestimando que la citada modificación del Plan General que levantaba la protección integral del hospital pudiese ser tenida en cuenta, pues se había hecho con posterioridad a la sentencia que obligaba a su derribo $^{16}$. Es significativo, además, que una de las últimas decisiones judiciales ${ }^{17}$, y que confirma el derribo, recurriese al peritaje de la Real Academia de Bellas Artes de San Fernando, que emitió un durísimo informe que aun valorando positivamente la localización de la Facultad en la Alcazaba, al considerarlo un uso noble con posibilidades de ayudar a la revitalización de la zona alta de la ciudad, lamentaba "la baja calidad del proyecto, tanto en el orden conceptual, como en el material y constructivo" 18 .Pese a haber pasado más de un año desde su emisión, la sentencia de 2012 del T.S.J. de Extremadura no se ejecutó, al presentar el Ayuntamiento un recurso de casación en el que defendía la imposibilidad de aplicarla.

${ }^{16}$ Esta misma estrategia se había seguido para derribar la vieja plaza de toros y edificar un palacio de congresos junto a uno de los baluartes de la muralla Vauban, pero en este caso la modificación fue anterior a la concesión de la licencia de derribo (LóPEZ-LAGO, 2009).

${ }^{17}$ El auto del TSJ de Extremadura de 24 de abril de 2012 puede consultarse en http://servicios.hoy.es/datos/documentos/auto-cubo-biblioteconomia.pdf

18 Informe de la Real Academia de Bellas Artes de San Fernando http://servicios.hoy.es/ datos/documentos/informe-cubo-real-academia.pdf

Polígonos. Revista de Geografía, 25 (2013); 57-89 
Sin embargo, en el momento en que se redactaban estas líneas dicho recurso ha sido finalmente desestimado ${ }^{19}$, por lo que debería ejecutarse eliminado las partes del edificio señaladas en la anterior sentencia del T.S.J. de Extremadura.

Desde el punto de vista urbano la discusión se centra en dos aspectos fundamentales. Por un lado, el supuesto coste económico del derribo, monto a asumir por el Ayuntamiento como otorgante de la licencia, y que algunas fuentes sitúan aproximadamente en los ocho millones de euros, lo cual es rebatido por los partidarios de la ejecución ${ }^{20}$. En segundo lugar, se plantearía la imposibilidad, señalada por el decano de la Facultad, de seguir desarrollando en ese edificio la actividad educativa y científica propia de esta institución en caso de perder los espacios que deberían demolerse (despachos de profesores, un salón de actos y parte del aulario); resultado no deseado por la mayoría de los defensores de la restauración de la situación originaria, en la medida en que se oponen a la solución técnica pero no a la ideológica, la localización de la función universitaria en la Alcazaba.

Imagen 2.Montaje publicado en el Diario Hoy con la vista actual de la Alcazaba y la torre de Espantaperros, y la situación final una vez se ejecutase la sentencia.

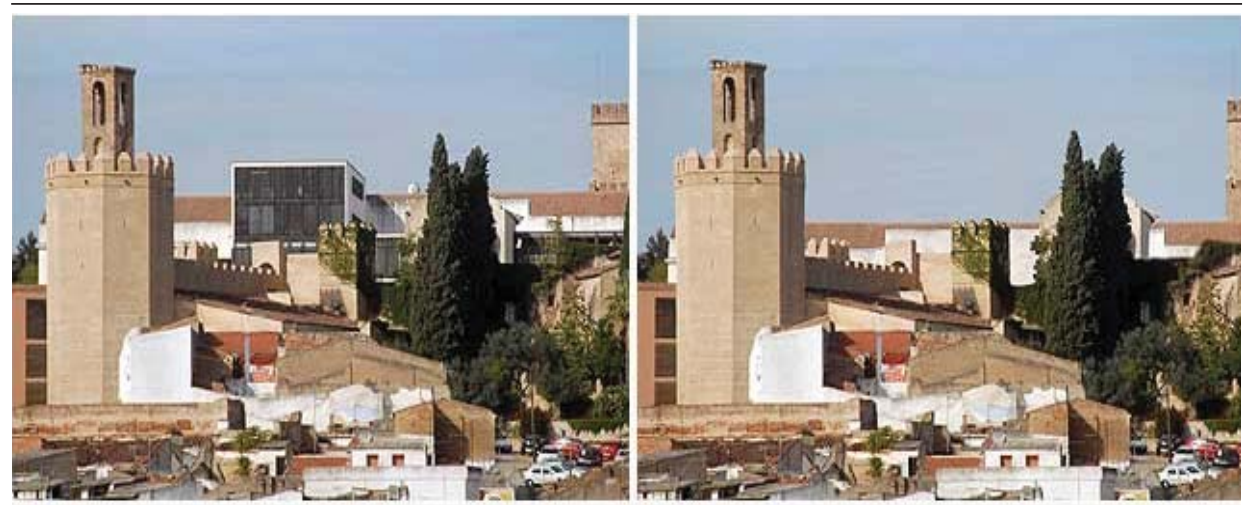

FuENTE: http://www.hoy.es/20091113/local/badajoz/fragoso-voluntad-ciudad-dice-200911 131404.html.

Es en esta disyuntiva, la aparente contradicción entre restaurar el perfil de la Alcazaba y continuar con la función universitaria, en donde se pueden centrar las conclusiones más importantes en relación con el marco teórico construido

${ }^{19}$ Sentencia desestimatoria de la Sección Quinta de la Sala de lo Contencioso - Administrativo del Tribunal Supremo, del 10/10/2013.

${ }^{20}$ Así lo señalaron tanto el representante político de IU entrevistado, Manuel Sosa, como el arquitecto Javier Teijeira, perito de Amigos de Badajoz. 
al inicio de este texto. En efecto, y al margen de los mayores o menores costes económicos, el problema se plantea en cómo encontrar viabilidad técnica a un conflicto en torno a una intervención en la que existiendo un casi total acuerdo ideológico, sobre la función y su localización, parece que nunca hubo una verdadera voluntad de hallar un consenso técnico, sobre cómo llevarla a cabo en lo urbanístico y lo arquitectónico. Es decir, compaginar la protección de un patrimonio de gran valor y de un enorme simbolismo para la ciudad de Badajoz (disenso técnico) con los intentos de recuperación y rehabilitación de la parte alta del casco histórico (consenso ideológico) a través de funciones elevadas como son las universitarias ${ }^{21}$.

\section{IV.2 La inserción arquitectónica y simbólica del hotel Atrio Relais \& Châteaux en la ciudad monumental de Cáceres}

La historia de la moderna protección del patrimonio urbano de Cáceres comenzó en 1930 con la declaración como BIC de las murallas de la ciudad, a las que se sumaron tan solo un año después varios de los inmuebles más significativos, y finalmente la totalidad del conjunto en 1949. Este proceso de protección del patrimonio urbano cacereño alcanzó un rango internacional cuando el Consejo de Europa lo catalogó como Tercer Conjunto Monumental de Europa (1968), y puede darse por finalizado en 1986 cuando la UNESCO inscribió a la ciudad en la Lista del Patrimonio Mundial. Este alto nivel de institucionalización, junto con el carácter que ese núcleo genético confiere al conjunto de la ciudad (CAMPESINO, 1982), pese a que en la actualidad haya perdido su papel de centro polarizador de la vida urbana, explica la transcendencia que pueden llegar a presentar algunas intervenciones en el mismo, especialmente si implican la sustitución de elementos preexistentes y simbólicamente consolidados, por más que no tengan gran valor patrimonial, por formas y lenguajes contemporáneos.

Un buen ejemplo en este sentido es la construcción del hotel Atrio Relais \& Châteaux en dos parcelas situadas entre la plaza de San Mateo y las calles de los Condes y del Olmo, en la parte alta de la ciudad amurallada; la primera de ellas, ocupada por un falso histórico construido en 1981, fue comprada por los promotores del hotel, mientras que la segunda era propiedad de la Junta de Extremadura. El comple-

${ }^{21}$ Debe señalarse que si bien la mayor parte de los entrevistados estaban de acuerdo con la instalación de la Facultad en la Alcazaba como un elemento importante para la recuperación del casco, este acuerdo que se ha calificado de ideológico no es absoluto. El arquitecto Javier Teijeiro manifestó en la entrevista realizada su oposición no solo al proyecto arquitectónico sino también a la función, señalando que la Alcazaba es un espacio arqueológico y debería tratase como tal, sin incluir nuevas funciones $y$, por supuesto, sin construcciones de nueva planta. 


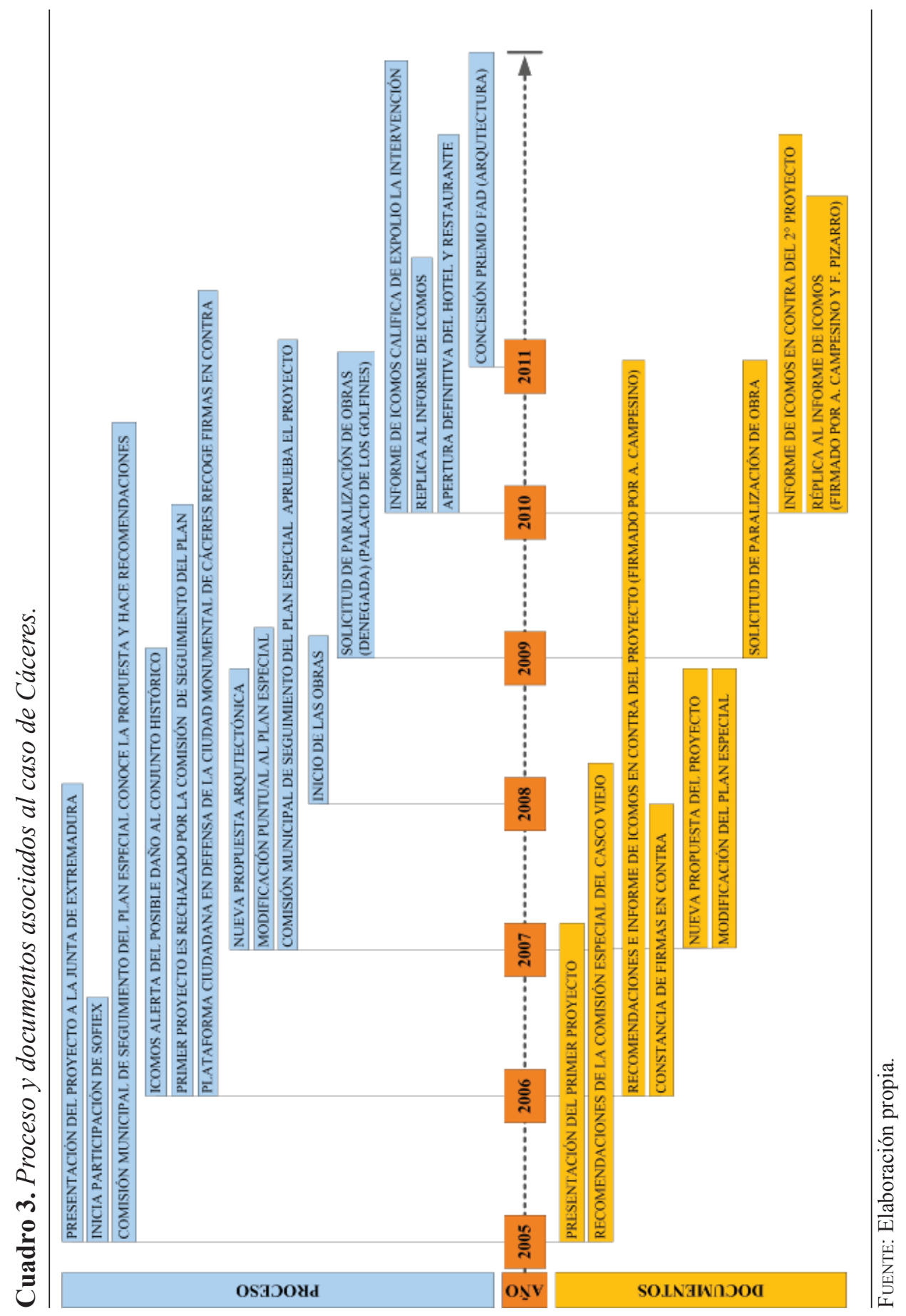

Polígonos. Revista de Geografia, 25 (2013); 57-89 
jo proceso de definición del proyecto, que aparece sistematizado en el Cuadro 3, dio comienzo cuando en enero del 2005 se presentó la idea por parte de los promotores a la Junta de Extremadura, que anunció la participación de la Sociedad de Fomento Industrial de Extremadura (SOFIEX) con un total de 9,6 millones de euros, más la cesión de la parcela pública arriba citada situada en la plaza de San Mateo nº 3 .

Tras unas primeras recomendaciones de modificación por parte de la Comisión Municipal de Seguimiento del Plan Especial en ese mismo año, el gobierno local intentó presentarlo de nuevo ante dicha comisión a pesar del visado negativo por parte del Colegio de Arquitectos de Cáceres, con el subterfugio de considerarlo “intervención singular". Es entonces, en el año 2006, cuando se inicia la verdadera oposición pública al proyecto por parte de la denominada Plataforma Ciudadana en Defensa de la Ciudad Monumental de Cáceres (PCMC), formada por asociaciones de vecinos de la ciudad monumental y de ecologistas y de defensa del patrimonio como ADENEX, así como algunas personalidades independientes pero con un significativo reconocimiento académico y técnico, entre los que cabe destacar al catedrático de la Universidad de Extremadura Antonio Campesino, redactor del primer informe de ICOMOS contrario al proyecto ${ }^{22}$. Dicho movimiento opositor se vio fuertemente impulsado cuando se conocieron las primeras imágenes del proyecto presentado por los prestigiosos arquitectos Emilio Tuñón y Luis Moreno Mansilla (Imagen 3), lo que motivó desde la recogida de firmas hasta manifestaciones en contra del mismo. La Comisión Municipal de Seguimiento del Plan Especial rechazó el proyecto en marzo de 2006, rechazo que fue ratificado por un Ayuntamiento que en principio se había mostrado a favor del mismo, rompiendo así el consenso político que hasta ese momento mantenía con la Junta de Extremadura.

Imagen 3. Conjunto de edificaciones preexistentes y montaje de la PCMC según el primer proyecto presentado por Tuñón y Mansilla.

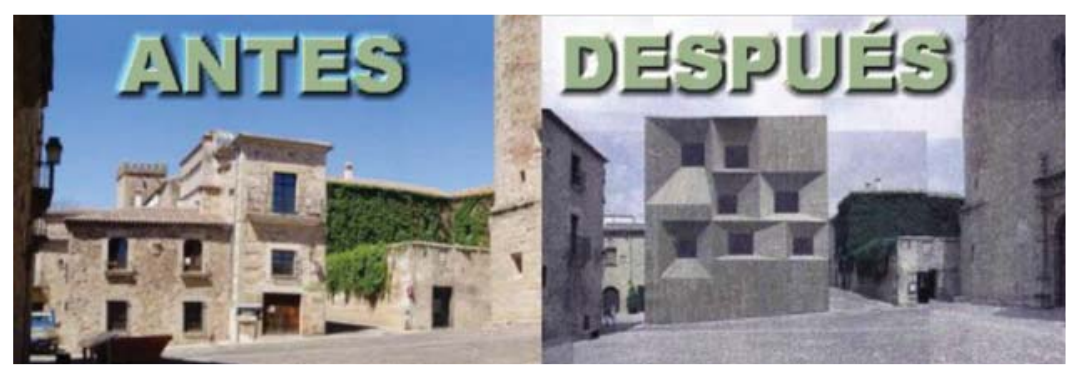

FUENTE: Extraído del pliego de firmas contra el proyecto presentado por la PCMC.

${ }^{22}$ ICOMOS. Informe sobre intervención arquitectónica - urbanistica en la Ciudad Vieja de Cáceres, Patrimonio Mundial. Proyecto Atrio Hotel Relais \& Châteaux en la Plaza de San Mateo (Cáceres). Firmado por A. Campesino en febrero de 2006. 
A partir de ese momento se produjo un cambio significativo en el proceso, dado que el equipo de arquitectos se puso en contacto con Antonio Campesino, una de las cabezas visibles de la oposición, y le requirieron su colaboración para la elaboración de un nuevo proyecto que encajase en la planificación urbanística y especial de Cáceres ${ }^{23}$. Se inicia, de este modo, una fase de colaboración entre algunos de los principales agentes a favor y en contra, que según la información recogida, y de acuerdo con la estructura interpretativa desarrollada en la introducción de este texto, se situó esencialmente en el plano técnico urbanístico.

En efecto, y aunque gran parte de la controversia ciudadana vino motivada por una mezcla de cuestiones funcionales y técnicas, como las características estéticas de la propuesta y el impacto sobre el simbolismo y el paisaje de la ciudad intramuros ${ }^{24}$, la oposición se concretó técnicamente en el incumplimiento de la legalidad del entonces vigente Plan Especial de Protección y Revitalización del Patrimonio Arquitectónico de la Ciudad de Cáceres (PEPRPACC) de 1990 en cuanto a uso, volúmenes, huecos y materiales. El resultado de la referida colaboración entre actores fue la presentación de una nueva propuesta arquitectónica en enero de 2007, que solventaba en parte este conjunto de problemas, pero que además, aunque directamente motivado por lo anterior, conllevaba también un radical cambio estilístico, tal y como puede observarse en la Imagen 4. De todos modos, el nuevo proyecto requirió una modificación del PEPRPACC de 1990 en cuanto a la autorización para el cambio de uso, que pasó de residencial e institucional a hotelero, y para la agregación de las dos parcelas en una sola ${ }^{25}$.

${ }^{23}$ De acuerdo con la información facilitada por Antonio Campesino, la iniciativa de la colaboración debió partir directamente de los arquitectos más que de los agentes económicos impulsores, los conocidos empresarios propietarios del restaurante Atrio. Esto no pudo ser corroborado en la entrevista realizada con los inversores, dados los problemas señalados en el tercer epígrafe.

${ }^{24}$ Debe hacerse especial hincapié en dicho impacto paisajístico, tanto a nivel intraurbano como desde el exterior. En cuanto al primero, y al margen de las características estéticas, el volumen ocultaba desde la plaza de San Mateo algunos elementos de gran valor, como la torre del Palacio de los Golfines. En cuanto al impacto desde fuera, la altura del edificio modificaba el perfil de la ciudad histórica vista desde las rondas o desde el Santuario de la Montaña, tal y como defendieron los integrantes de ADENEX entrevistados.

25 Dicha modificación se aprobó a pesar de que el artículo 41.2. de la Ley 2/1999, de 29 marzo, de Patrimonio Histórico y Cultural de Extremadura señala: "Se mantendrán igualmente la estructura urbana y arquitectónica del Conjunto Histórico y las características generales del ambiente y del paisaje. No se permitirán modificaciones de las alineaciones, alteraciones de la edificabilidad, parcelaciones ni agregaciones de inmuebles, salvo que contribuyan a la conservación general del carácter del Conjunto Histórico". 
Esta segunda propuesta recibió el visto bueno de la Comisión Municipal de Seguimiento del Plan y de la Comisión de Urbanismo ese mismo año, iniciándose las obras en marzo de 2008. Sin embargo, y a pesar de este relativo acuerdo entre parte de los actores antes contrarios, siguieron existiendo importantes controversias, producidas cuando la construcción del hotel se encontraba muy avanzada.

La primera fue la denuncia en 2009, por parte de los propietarios del cercano palacio de los Golfines de Arriba, por un posible delito contra dicho edificio, que fue finalmente desestimada. En segundo lugar, y con una crítica de mayor trascendencia, se hizo público un informe presentado en julio de 2010 por ICOMOS, calificando de "expolio" la intervención que en ese momento se estaba desarrollando, y apuntando como principales argumentos la no conservación de un conjunto de bóvedas protegidas, la desagregación de parcelas y la supuesta rebaja de la calificación de "ambiental singular" que los edificios preexistentes tenían de acuerdo con el PEPRPACC. Dicho informe fue rápidamente contestado por otro firmado por los profesores de la Universidad de Extremadura Antonio Campesino y Francisco Pizarro, miembros de ICOMOS en la ciudad de Cáceres, en donde sostenían que no se producía dicha desagregación, sino antes al contrario una agregación de parcelas que devolvía a la situación anterior a 1980, cuando se construyó uno de los inmuebles preexistentes; y que ninguna de las dos parcelas previas tenía en el PEPRPACC la calificación de "ambiental singular" sino simplemente de "ambiental", lo cual era además compatible con la función hotelera ${ }^{26}$.

La apertura final de un hotel de lujo, cinco estrellas con catorce habitaciones, y del restaurante se produjo en diciembre de 2010, y el edificio recibió el Premio FAD de arquitectura en el 2011. Si bien, como se verá más adelante, siguió existiendo una importante oposición al mismo por parte de diversos grupos, la realidad es que una vez finalizado la presión social se redujo de forma notable, y poco a poco fue siendo aceptado por algunos de los sectores que en un inicio se habían opuesto.

${ }^{26}$ El Informe sobre la realización de un hotel en la Ciudad Vieja de Cáceres Patrimonio Mundial de ICOMOS puede consultarse en http://www.hoy.es/apoyos/documentos/informeicomos2.pdf; y la respuesta de los citados profesores, Réplica al informe de ICOMOS sobre el Hotel Atrio, en http://servicios.hoy.es/datos/documentos/replica-sobre-atrio.pdf. Debe tenerse en cuenta que A. Campesino había firmado el primer informe de ICOMOS en contra del primero de los proyectos presentados.

Polígonos. Revista de Geografía, 25 (2013); 57-89 
Imagen 4. Hotel Atrio desde la Plaza de San Mateo, y detalle de la pérgola y techumbre objeto de las críticas más importantes desde el punto de vista técnico tras la finalización del edificio.
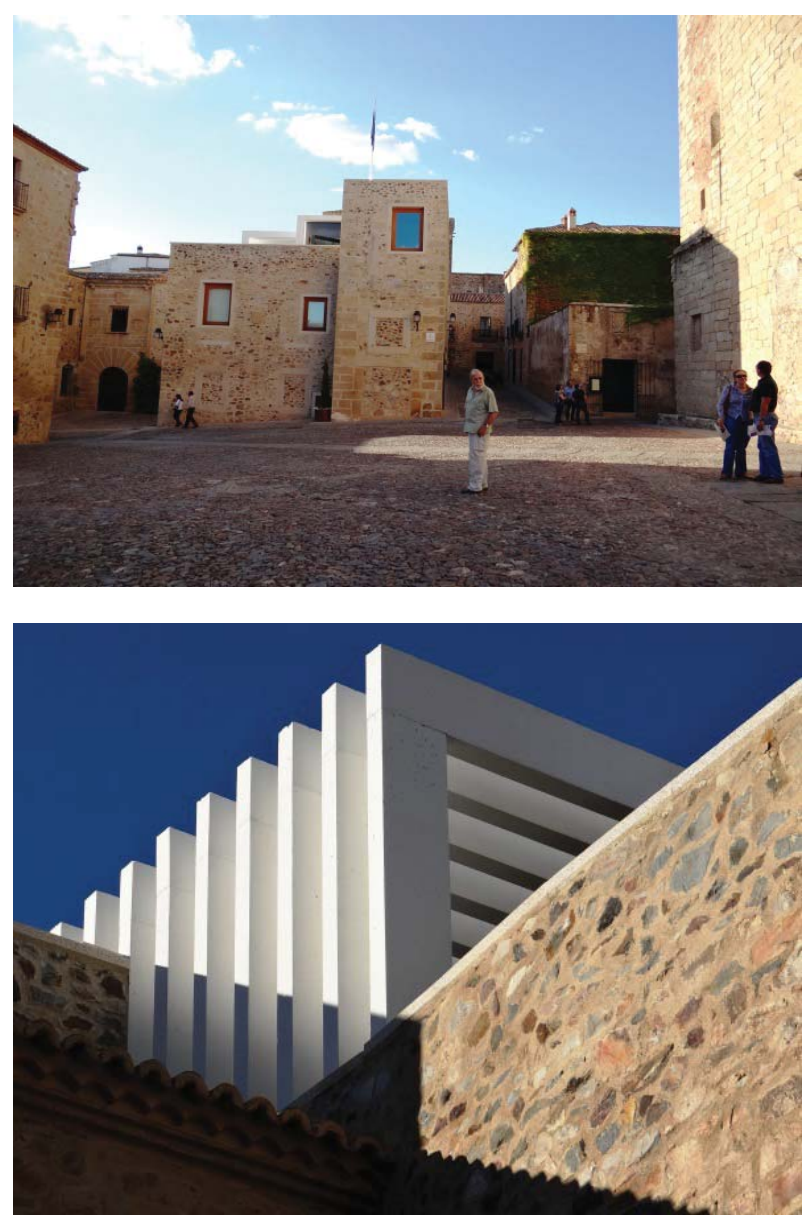

FuENTE: David Porras Alfaro. 


\section{ACTORES, NEGOCIACIÓN E INFLUENCIA. BASES PARA UNA INTERPRETACIÓN SOBRE LAS POSICIONES IDEOLÓGICAS Y TÉCNICAS}

En el Cuadro 4 se han sistematizado los agentes institucionales, económicos, profesionales y sociales que participaron en cada uno de los dos estudios de caso analizados, señalando tanto la influencia y posiciones que mantuvieron como los posibles cambios de postura producidos durante el proceso de conflicto y negociación, en función de las modificaciones introducidas o de los resultados que se fueron obteniendo ${ }^{27}$. El objetivo del análisis que se va a realizar en este epígrafe resulta esencial en relación con las hipótesis y el marco interpretativo explicitado, y que trata de mostrar cómo las relaciones de consenso y de disenso se pueden establecer a distintos niveles, en lo ideológico y en lo técnico; y cómo del papel representado en cada uno de estos aspectos y de la influencia obtenida por los distintos agentes se derivan muy diferentes situaciones en cuanto a la aceptación final del proyecto en lo funcional, lo simbólico, lo arquitectónico y lo urbanístico.

Se quiere recalcar lo que con anterioridad se calificó de carácter oportunista de los proyectos, y su escasa relación con estrategias más amplias y claramente explicitadas de tratamiento de la ciudad histórica, derivadas de la planificación o de planteamientos de carácter omnicomprensivo. Esto supone, además de la no definición de una visión amplia sobre el papel de la ciudad heredada en el conjunto urbano, eliminar o limitar la posibilidad de participación de la sociedad a través de los sistemas legalmente regulados en el planeamiento.

Tal y como pusieron de manifiesto varios de los entrevistados, en los dos casos el impulso institucional parte de la administración regional, lo que resulta mucho más significativo en el ejemplo cacereño en la medida en que se trata no de un servicio público educativo como en Badajoz, sino de una actividad económica de carácter privado. Los propietarios del hotel Atrio ya disponían de un restaurante de gran prestigio ${ }^{28}$ con el mismo nombre, pero localizado fuera de la ciudad histórica, y por tanto, sin conexión física con el simbolismo cultural y turístico de Cáceres. De acuerdo con la información obtenida en la entrevista con

${ }^{27}$ Con el fin de interpretar correctamente el cuadro debe tenerse en cuenta que los cambios de postura señalados con flechas pueden indicar dos situaciones diferentes. Cuando la flecha se acompaña con un cambio del agente a la otra columna se indica que el conjunto de la institución cambia de postura, siendo la segunda y definitiva la situada en el destino de la flecha; sin embargo, cuando solamente se muestra la flecha se indica que solo una parte de los integrantes de dicho actor institucional modificó su postura.

${ }^{28}$ Contaba con dos estrellas Michelin. 


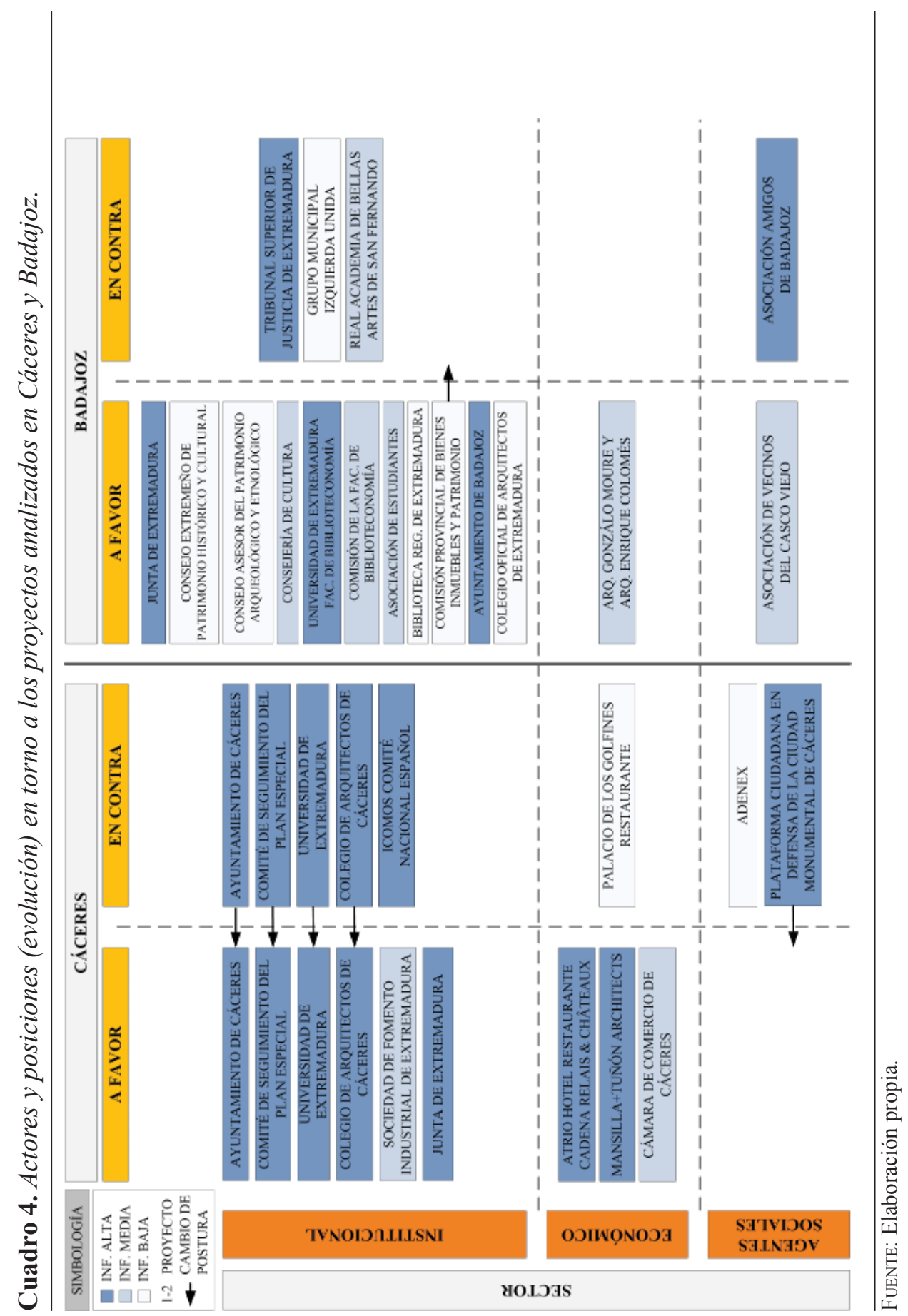


Antonio Campesino, gran parte del impulso inicial, y aparentemente también la elección de los arquitectos, pudo provenir de la propia Junta de Extremadura, que además participó a través de SOFIEX con una cantidad final que puede situarse en torno a los once millones de euros. La razón podría estribar en el intento del gobierno regional, aceptado en primera instancia por el local, de conectar el prestigio de la empresa con el simbolismo urbano ligado a la ciudad monumental; apostando, además, por un equipo de arquitectos que podrían entrar en el grupo de los denominados "estrella", y que previsiblemente proyectarían un edificio con un alto grado de capacidad icónica.

El segundo gran grupo de actores intervinientes en Cáceres estaba formado por profesionales y técnicos junto con asociaciones de vecinos y defensa del patrimonio y de los recursos, agrupados en la Plataforma Ciudadana en Defensa de la Ciudad Monumental de Cáceres (PCMC). Se establece, por tanto, una dualidad entre actores institucionales autonómicos y económicos frente a sociales, a favor y en contra del proyecto respectivamente. El papel de otros agentes con responsabilidades y competencias fundamentales, caso del gobierno local, queda relativamente diluido, ya que al asentimiento inicial le siguió su denegación una vez que la Comisión de Seguimiento del Plan se opuso, pero sobre todo, una vez que se constató la importante presión en contra que se había generado.

Tal y como se ha comentado con anterioridad, la oposición al primero de los proyectos presentados en Cáceres puso el acento en las cuestiones estéticas y en el impacto paisajístico. Es decir, la crítica se centró esencialmente en la concreción final del proyecto, lo que aquí se está considerando como una cuestión de disenso en los aspectos técnicos, mientras que quedaron fuera del principal foco de interés las cuestiones ideológicas, es decir, la discusión sobre la aceptación o no de la función hotelera de lujo y el papel real de la misma en la revitalización de la ciudad histórica. Así lo señaló Antonio Campesino en la entrevista realizada, donde incidió en que discutió con los arquitectos sobre el cumplimiento de la legalidad urbanística representada en ese momento por el PEPRPACC; y así se pone también de manifiesto en algunas entrevistas realizadas a los arquitectos Luis Moreno Mansilla y Emilio Tuñón, en las que se reconocía el error técnico del primer proyecto pero se incidía en la validez de este tipo de soluciones para la revitalización del casco, apuntando que "después de una primera propuesta fallida, realizada con independencia de la ciudadanía, el segundo proyecto presentado (...) fue realizado a través de un fluido y enriquecedor diálogo con los ciudadanos, las fuerzas vivas y las instituciones (...) con el firme propósito de participar en la revitalización de un lugar concreto del casco histórico"29.

${ }^{29}$ Hostelpro. http://www.doopaper.com/pubs/hostelpro/hostelpro1/?p=80. 
Se quiere resaltar que asociaciones como ADENEX, integradas en la PCMC y que se mantuvieron contrarias al proyecto tras su modificación, apuntaban, además de a los aspectos técnicos, a planteamientos ideológicos, al cuestionarse el posible papel de un hotel de lujo en la revitalización; así como a señalar el agravio comparativo que suponía para los residentes el cumplimiento de todos los requerimientos de un plan que, sin embargo, se modificaba para acoger al hotel. Mantenían en este sentido, y seguían manteniendo en la entrevista realizada, una crítica a la terciarización elitista de los cascos históricos en contraposición con la residencia, el comercio y los servicios de cercanía, así como a la supuesta monumentalidad y capacidad simbólica de la nueva arquitectura. Sin embargo, la realidad es que no fueron capaces de llevar al primer plano de la discusión pública esa otra perspectiva del problema, una vez que una parte significativa de los agentes y del conjunto de la sociedad aceptó la solución técnica presentada como segundo proyecto.

En el caso de Badajoz la oposición se sitúa entre actores institucionales, con una mayor importancia del gobierno local que concedió la licencia, que ha figurado en el primer plano mediático y sobre el que se han centrado la mayor parte de las críticas; frente a sectores sociales conservacionistas, representados por Amigos de Badajoz. Otras asociaciones, como la de residentes en el casco viejo o la de comerciantes, han apoyado el proyecto, pero sin tener en ningún momento un papel que pueda ser calificado de protagonista.

Al contrario de lo que sucedió con el proyecto hotelero de Cáceres, en el caso de Badajoz existía de partida un casi total acuerdo en lo ideológico ${ }^{30}$, es decir, en torno a la idoneidad de la función universitaria como una actividad adecuada para la revitalización del casco histórico, y de forma expresa para cambiar la negativa imagen presentada por la parte alta del mismo, de la que había desaparecido cualquier servicio de escala urbana. Sin embargo, y a pesar de que varios de los entrevistados pusieron de manifiesto la realización de numerosas reuniones que casi podrían ser calificadas de secretas, en ningún momento se consiguió llegar a un acuerdo que aportase una solución técnica a lo que para gran parte de los agentes era una buena idea.

Lo que se produjo fue una escalada de enfrentamientos, llegando en ocasiones a lo que podría ser catalogado como descalificaciones personales ${ }^{31}$, estas

${ }^{30}$ Ya se señaló la excepción que entre los entrevistados suponía Javier Teijeiro, arquitecto y perito de la Asociación Amigos de Badajoz.

31 El entonces alcalde de Badajoz señalaba a la prensa en el 2010 que la insistencia de Amigos de Badajoz denotaba "no amor a Badajoz sino «mala leche»" (sic) (Romero, 2010). 
poco a poco fueron haciendo cada vez más complicado el hallazgo de vías de solución técnica. De hecho, de las manifestaciones en prensa se deriva que cada una de las partes percibía a la otra de un modo exclusivamente negativo, bien como representantes de un conservacionismo retrógrado o de la prepotencia técnico - política.

Como ya se ha comentado en el apartado de fuentes, no fue posible realizar la entrevista con uno de los agentes más importantes, el Ayuntamiento de Badajoz, lo cual puede influir en la valoración que aquí se está haciendo. En todo caso, de la información publicada parece desprenderse que tanto el gobierno local como el equipo de arquitectos parecían confiar en que, bien derivado de la supuesta calidad arquitectónica del proyecto, o bien del agotamiento por la duración del conflicto, los opositores acabarían finalmente aceptando el resultado, o al menos renunciando a la restitución de la situación original en función de una política de hechos consumados. Así, en una entrevista realizada durante la construcción, los arquitectos pedían "que se deje terminar el conjunto arquitectónico (...) para que este alcance toda su significación (...) seguros de que la polémica se agotará en cuanto el edificio se adapte a sus nuevas funciones" (BARRADO, 2000); mientras que la entonces concejala de urbanismo, tras la decisión tomada en 2000 por el Ayuntamiento y la Junta de continuar con el proyecto original a pesar de la denuncia presentada, apostaba por "esperar a que termine la obra del edificio de nueva planta para saber si verdaderamente causa impacto visual (...), y al final siempre se puede optar por derribar una planta si así se cree conveniente" (sic) (Cid DE Rivera, 2000).

Podría concluirse señalando que en Cáceres se consiguió integrar un conjunto de actores - eje representantes de cada una de los dos posturas enfrentadas; por un lado, y opuesto al proyecto, el catedrático Antonio Campesino, y en el otro, a favor del mismo, el equipo de arquitectos Tuñón y Mansilla. También fue definitivo el papel del Colegio de Arquitectos de Cáceres, clave en la articulación de las soluciones como ámbito físico e intelectual de discusión entre posiciones opuestas, y que como institución no se situó en una postura de defensa corporativa de los arquitectos como el único grupo supuestamente cualificado para afrontar el debate ${ }^{32}$. Este acuerdo se produjo, como ya se ha señalado, en los aspectos técnicos, y conllevó la modificación del PEPRPACC y finalmente la concesión de visado y licencia al proyecto.

${ }^{32}$ De hecho, el Colegio de Arquitectos de Cáceres visó negativamente el primero de los proyectos presentados, alegando el incumplimiento normativo en cuanto a alineaciones, volúmenes, edificabilidad, materiales y texturas, y ratificando en gran medida el razonamiento realizado por el geógrafo A. Campesino en el ya citado informe que redactó para ICOMOS en febrero del 2006. 
Esto no obsta para que siguiese existiendo desacuerdo ideológico, pero que ya no se tradujo, como antes había sucedido, en un rechazo frontal a la materialización del nuevo edificio. De hecho, los grupos opositores entrevistados, y el informe crítico de ICOMOS de 2010 al que ya se hizo mención, centran el disenso técnico en un aspecto que podría considerarse menor, como es la no conservación de un conjunto de bóvedas que a su vez ya estaban muy modificadas; mientras que mantienen la oposición ideológica, más o menos matizada, a la introducción en la ciudad histórica de un lenguaje arquitectónico con una cierta pretensión de iconicidad.

Sin embargo, en el caso de Badajoz no fue posible encontrar eso que arriba se calificó de actores - eje, que consiguiesen articular una respuesta técnica adecuada a ese casi total consenso ideológico que existía de partida. Quizá la aproximación de posiciones podría haberse producido entre la Facultad y la Asociación Amigos de Badajoz, agentes que en las entrevistas manifestaron haber asistido a varias reuniones multilaterales. Sin embargo, y a pesar de que ambas instituciones contaban con técnicos de diverso tipo (historiadores, expertos en patrimonio, urbanistas, arquitectos, etc.), no consiguieron alcanzar un acuerdo.

En todo caso, debe resaltarse que Amigos de Badajoz planteó desde muy pronto lo que podía ser considerado como una oferta de solución técnica, no haciendo bandera de la restitución total de la situación inicial sino tan solo del perfil de la Alcazaba, para lo que bastaría con derribar los elementos de nueva planta que sobresalen sobre el perfil de la muralla; posibilidad que la Facultad considera no viable desde el punto de vista funcional, y que en caso de llevarse a cabo les obligaría a abandonar el edificio. Esta interpretación del interés de Amigos de Badajoz por consensuar un acuerdo en lo técnico que salve la función universitaria en la Alcazaba se pone de manifiesto por su expresa valoración positiva del resultado final obtenido con Atrio en Cáceres, y que su presidente Antonio Manzano puso como uno de los "buenos ejemplos de la Administración, que había corregido a tiempo antes de que se consumaran obras ilegales" (LóPEZ-LAGO, 2007).

En este sentido, quiere señalarse de forma negativa el nulo papel que la Universidad de Extremadura, al margen de la Facultad de Biblioteconomía, ha tenido como institución, y que podría haber coadyuvado a la búsqueda de soluciones a partir de su posición como agente y a su capacidad científica y técnica en el conjunto de materias relacionadas con el problema planteado. Este aspecto aparece expresamente recogido de forma muy crítica en el informe de la Real Academia de Bellas Artes de San Fernando, que pone de relieve como de acuerdo con la Ley de Patrimonio Histórico y Cultural se trata de una de las instituciones consultivas a los efectos previstos en dicha ley. 
Más allá de planteamientos personales, resulta complejo apuntar a las razones que permitieron a los diferentes agentes, a partir de una situación conflictiva de partida relativamente parecida, articular en un caso sí y en otro no un cierto nivel de consenso técnico. Seguramente la principal línea argumental deba avanzar por la muy diferente situación, catalogación y valoración que los respectivos conjuntos históricos tienen en cada una de las ciudades analizadas.

En el caso de Cáceres, como se vio al inicio de este texto, la ciudad antigua tiene los niveles más altos de catalogación, y es, sin ninguna duda, un elemento de enorme presencia positiva en lo simbólico y en lo económico, por más que presente importantes niveles de aislamiento social y territorial debido a la pérdida de la función residencial y comercial. En resumen, se trata de una pieza relativamente aislada pero bien valorada, entendida como un "bien para la ciudad" 33 . Por el contrario, el casco de Badajoz, aunque presenta numerosos elementos de evidente interés patrimonial, entre ellos la Alcazaba, es claramente un espacio vulnerable y degradado en el que se han puesto en marcha múltiples intentos de regeneración y rehabilitación que la mayoría de los agentes catalogaron de fracasados. Pero mucho más allá, aunque en los últimos años se haya producido algún pequeño avance, es sobre todo un área simbólicamente estigmatizada dentro del conjunto urbano, y físicamente rechazada por gran parte de los pacenses, que jamás suben a la parte alta.

Desde este punto de vista la ciudad histórica de Cáceres, o al menos la ciudad amurallada, es percibida como un área estabilizada y altamente institucionalizada en relación a sus niveles de protección, y por tanto, a las posibilidades de intervención y de innovación en cuanto a la introducción de nuevos lenguajes ${ }^{34}$. Esto conlleva la existencia de un cierto consenso respecto al grado de transformación que la sociedad puede admitir sin que se produzca una ruptura con la simbología y la imagen del conjunto, lo que permite reconducir técnicamente desacuerdos centrados en lo funcional.

Por el contrario, el casco de Badajoz es claramente un espacio en movimiento y discusión, necesitado de una clara redefinición. Más allá de la asunción de que es necesario hacer algo, lo que permitiría acuerdos básicos en la introduc-

33 Esta calificación de "bien para la ciudad" referido a la ciudad histórica de Cáceres se la debemos a Ángela Matesanz, arquitecta e investigadora del Departamento de Urbanismo de la Universidad Politécnica de Madrid. Sugirió esta línea de interpretación en una presentación de las primeras conclusiones de esta investigación en el marco de las VI Jornada de Investigación Urbana - Proyecto URBSPAIN, celebradas en la Universidad Autónoma de Madrid el 11 de julio de 2013.

${ }^{34}$ Esto no implica que la historia de la protección e institucionalización de la ciudad histórica no sea ni conflictiva ni compleja, tal y como puede verse en A. Campesino (2009). 
ción de funciones que se considerasen nobles, es un ámbito de conflicto entre grupos sociales y económicos, entre visiones de la ciudad y de las formas de intervenir en la misma, así como en cuanto al papel que esta pieza debe representar en el conjunto urbano. Y si de la percepción de la degradación se puede derivar un cierto acuerdo ideológico sobre la necesidad de intervenir, de la multiplicidad de ámbitos de conflicto se deriva la dificultad de alcanzar acuerdos sobre cómo hacerlo.

\section{VALORACIÓN AMBIENTAL Y SOCIAL DE LOS PROYECTOS: CONFLICTO FRENTE A SOSTENIBILIDAD}

Uno de los objetivos de esta investigación, integrada en un proyecto más amplio referenciado al principio del texto, era avanzar en la definición de instrumentos que permitiesen juzgar desde un punto de vista social y ambiental determinadas intervenciones urbanas. Para ello se definió un conjunto de criterios con el fin de ser evaluados por los investigadores en función de las diversas fuentes, pero esencialmente a partir del trabajo de campo y de la percepción de los agentes entrevistados. Al margen de la valoración de los aspectos metodológicos, que se realizará en el marco conjunto del proyecto y en función de otras aproximaciones paralelas, se van a apuntar aquí, sin pretensión de exhaustividad, los aspectos que se consideran más significativos en cuanto a los casos analizados.

En primer lugar, señalar la valoración claramente negativa de ambos proyectos en cuanto a su integración y transversalidad, en la medida en que, como se apuntó más arriba, no derivan de estrategias integradas que respondan a una idea social y técnicamente consensuada sobre la ciudad histórica y su papel como pieza integrada en un continuo urbano. Avanzar en la definición de dichas estrategias permitiría mantener discursos coherentes desde el punto de vista ideológico, que no se encontrasen al albur de lo que aquí se ha calificado como proyectos oportunistas que pueden ser aceptados o rechazados a partir de aspectos coyunturales.

Por el contrario, se valoran de manera muy diferente dos criterios directamente conectados con el anterior, como son los de colaboración y gobernanza. En Cáceres se consiguieron acuerdos que si bien no pueden ser vistos positivamente desde el punto de vista de la gobernanza, al menos se sustanciaron a partir de procesos de coordinación que integraron a un porcentaje significativo de los agentes, algo que en ningún momento sucedió en Badajoz. Desgraciadamente, el caso de Badajoz solo puede ser visto desde estas perspectivas de manera negativa, en la medida en que la participación no dio lugar a la definición de un proyecto mayori- 
tariamente aceptado; problema que no fue posible sustanciar a partir de procesos de colaboración, y que ha acabado con una sentencia judicial, aún no ejecutada, que obliga a derribar parte del edificio.

El análisis de la capacidad de innovación es el único aspecto en el que el caso de Badajoz presenta una valoración similar o superior al de Cáceres, en la medida en que se considera posible que tenga una repercusión más positiva, en cuanto a la integración del casco en la dinámicas ciudadanas, la instalación de un servicio público elevado, como es una facultad en lugar de un hotel de lujo. Señalar, en este sentido, la dirección opuesta que presentan las últimas tendencias del urbanismo en cada una de las ciudades, pues mientras que en Badajoz se apuesta por integrar la universidad en la ciudad histórica, en Cáceres se optó por trasladar a un campus periférico y segregado las facultades y algunos servicios universitarios que se localizaban en la ciudad histórica, con ejemplos significativos como la Facultad de Derecho o la Escuela Politécnica ${ }^{35}$.

En cuanto a la repercusión en la imagen urbana, puede ser analizada en función del proceso o del resultado final. En ambos aspectos la valoración del hotel Atrio es positiva, pues se transmitió una imagen de acuerdo tras el conflicto, y el resultado final implica la dotación de un equipamiento hotelero diferenciado, y con un prestigio evidente tanto en lo referido a la autoría arquitectónica como en cuanto a los servicios ofrecidos. Justo al revés parece haber sucedido en Badajoz, dado que ni desde el punto de vista arquitectónico y urbanístico, ni desde el procesual, pueden derivarse beneficios en imagen para la ciudad. Como se ha apuntado a lo largo del texto, en el caso de Cáceres se juega con la búsqueda de una nueva monumentalidad, como un intento de incrementar el capital simbólico de la ciudad, en lo que parece que se ha tenido un cierto éxito más allá de cómo se valoren ideológicamente estos intentos.

De hecho, la percepción final del impacto es moderadamente positiva en el caso de Cáceres y negativa en Badajoz. Eso no obsta para que, en cuanto a los avances alcanzados, la situación de un ejemplo y otro se matice notablemente, en la medida en que a pesar de todos los problemas comentados en el ejemplo pacense, prácticamente la totalidad de los agentes valoran de manera positiva la presencia de los estudiantes en la zona alta de la ciudad, por más que la mayoría señale también que de su mera presencia no se han derivado cambios a mejor en dicho ámbito.

${ }^{35}$ La evolución urbana y territorial de la Universidad de Extremadura en el semidistrito de Cáceres, con el proceso de transformación, ocupación y posterior abandono de numerosos edificios en el centro histórico de la ciudad, puede seguirse en A. Campesino (2007). 


\section{CONCLUSIONES Y ELEMENTOS PARA EL DEBATE FUTURO}

Del análisis de los estudios de caso en Cáceres y Badajoz se ha puesto de manifiesto como el conflicto y la posibilidad de acuerdo en torno a proyectos de intervención en ámbitos históricos puede establecerse a múltiples niveles. Se ha planteado como una vía de interpretación que estas diferentes capas de consenso y disenso pueden estar relacionadas con la percepción y el papel que los agentes, pero sobre todo que el conjunto de la sociedad, atribuyen a la ciudad heredada dentro del conjunto del espacio urbano, tanto desde el punto de vista simbólico como funcional. Avanzar en esta explicación obligaría a comparar ejemplos de intervención puntual, como los que aquí se han analizado, con otros en los que se valorase el papel de los diferentes agentes involucrados en proyectos que respondan a programas más amplios y omnicomprensivos sobre la ciudad histórica, redactados y aprobados a partir de procesos que admitiesen la reflexión y participación social.

La renovación urbana presenta una gran complejidad y dinámicas propias relacionadas con intereses políticos, sociales y económicos, que raramente podrán conjugarse adecuadamente a través de los modelos de participación instituidos en los sistemas de aprobación de los diferentes tipos de planeamiento. Además, debe diferenciarse claramente entre lo que sería la participación en torno a la realización de un plan o los ejemplos que aquí se han tratado, en relación al diseño de un proyecto concreto; es en estos casos de intervención puntual, que no están derivados de un plan previamente existente y redactado y aprobado de acuerdo con un proceso claro, en donde es más necesaria la transparencia que dé entrada a la participación ciudadana, tal y como señala la "Carta para la integración de la arquitectura contemporánea en las ciudades Patrimonio de la Humanidad" de Córdoba ${ }^{36}$.

En todo caso, de esta y otras experiencias, como las que han desarrollado diversos colectivos de urbanistas, puede derivarse que "a participar se aprende participando" (PAISAJE TRANSVERSAL, 2012), y que es factible acabar definiendo un conjunto de lo que aquí se ha denominado como actores - eje, con capacidad para definir soluciones de relativo consenso. No obstante, su articulación requeriría de mecanismos estructurados, que permitan llegar a acuerdos que apunten tanto a la conservación de los recursos culturales de la ciudad como a favorecer los procesos evolutivos a los que está sometida.

Debe admitirse que, sean cuales sean las formas de participación, la integración y aceptación social de nuevas funciones y lenguajes arquitectónicos

${ }^{36} \mathrm{http} / / / \mathrm{ww} w . f e m p . e s / f i l e s / 566-990$-archivo/Arq_contemporanea_ciudades_patrimonio.pdf. 
en los conjuntos históricos con un alto nivel de institucionalización se tornan difíciles. Pero a pesar de la dificultad que implica llegar a alcanzar consensos, se hace cada vez más latente su necesidad de aplicación. La inyección de vitalidad y la inserción de proyectos que de forma novedosa pongan en valor la ciudad y rehabiliten sus funciones permitirán su vigencia en el tiempo, y abrirán a su vez el espacio para nuevas actividades.

En todo caso, parece claro que a distintos niveles, y a partir de mecanismos complejos, los habitantes de una ciudad atribuyen a los espacios heredados un cierto nivel de simbolismo, una imagen y una idea relativamente estable sobre cuál debe ser su papel dentro del conjunto urbano. En este sentido, debe asumirse que cuando se interviene sobre estos ámbitos se pueden modificar dichos parámetros, lo que será mucho más conflictivo cuando se trate de incorporar proyectos con un cierto nivel de iconicidad, y que se superpongan tanto visual como simbólicamente sobre el patrimonio heredado.

\section{BIBLIOGRAFÍA}

Alvarez Mora, A. (2001): “La cuestión de los Centros Históricos: Generación de Planes y Políticas Urbanísticas recientes” en ALVAREZ, A. y CASTILLO, M. (Coords.), Ciudad, territorio y patrimonio: materiales de investigación: programa de doctorado enero 1999-diciembre 2000, Universidad de Valladolid, Instituto Universitario de Urbanística.

BARrado, M. (2000): "Los arquitectos de Biblioteconomía piden que se considere su proyecto de forma global". Diario Hoy de Extremadura, 15.09.2000.

Campesino, A. (1982): Estructura y paisaje urbano de Cáceres. Cáceres, Colegio de Arquitectos de Extremadura.

CAmpesino, A. (2007): “Ciudad y universidad: Cáceres, del campus patrimonial al ghetto montaraz", ATENEO. Revista científica, literaria y artística del Ateneo de Cáceres: Cáceres, Ateneo de Cáceres, 40 - 55.

Campesino, A. (2009): "Cáceres: gestión reciente de una ciudad del patrimonio mundial (1986 - 2008)", Ponencia en Simposio Internacional Ciudad sobre Ciudad (12 al 14 de noviembre de 2009): Valladolid, Fundación del Patrimonio Histórico de Castilla y León, 293 - 325.

CAstrillo, M. y JimÉnez, M. (2011): "La práctica de la arquitectura contemporánea en las ciudades históricas españolas. Notas para una aproximación histórico - urbanística", UNED, Espacio, tiempo y forma, Serie VII, Historia del Arte, t. 24, $291-322$.

Cid DE RiverA, A. (2000): “Junta y Ayuntamiento acuerda seguir adelante con las obras de Biblioteconomía”, Diario Hoy de Extremadura, 30.06.2000. 
GonzÁlez González, J. M. (2006): La rehabilitación de la Plaza Alta de Badajoz. Cáceres, Universidad de Extremadura.

Hernández Aja, A. et al. (2011): "Análisis urbanístico de barrios vulnerables. Badajoz", en Ciudades para un futuro más sostenible. Madrid, Universidad Politécnica de Madrid. Disponible en: http://habitat.aq.upm.es/bbvv/bbvv_112.html

López-Lago, J. (2007): “El cubo: 'Una solución quiero'”. Diario Hoy de Extremadura, 21.11.2007.

López-Lago, J. (2009): "La ajetreada historia del cubo". Diario Hoy de Extremadura, 22.11.2009. En línea: http://www.hoy.es/20091122/badajoz/ajetreada-historiacubo-20091122.html.

Pagés, J. M. y CAmpesino, A. (Dirs.) (1990): Plan especial de protección del patrimonio urbano de Badajoz. Memoria informativa, mecanografiado, 206.

Paisaje Transversal. (2012): “A participar se aprende participando. VdB Acción y reflexión críticas en el proyecto para la regeneración urbana participativa en el barrio Virgen de Begoña (Madrid)". Hábitat y sociedad, n ${ }^{\circ} 4$, mayo, 15 - 31.

RIVAS, DE LAS J. L. (2009): “Ciudad sobre ciudad. Interferencias entre pasado y presente urbano en Europa", Ponencia en Simposio Internacional Ciudad sobre Ciudad (12 al 14 de noviembre de 2009): Valladolid, Fundación del Patrimonio Histórico de Castilla y León, $19-37$.

Romero, R. (2010): “La insistencia de Amigos de Badajoz denota 'mala leche', dice Celdrán", Diario Hoy de Extremadura, 28.01.10. Disponible en: http://www.hoy. es/20100128/badajoz/insistencia-amigos-badajoz-denota-20100128.html

Troitiño, M. y GarcíA, J. (Coords.) (1998): Vivir las ciudades históricas: recuperación integrada y dinámica funcional. Cuenca, Ediciones de la Universidad de Castilla-La Mancha y Fundación La Caixa.

Agradecimientos: Los autores quieren mostrar su agradecimiento a los entrevistados por su participación durante el trabajo de campo, dichas aportaciones han sido fundamentales para la elaboración de esta investigación. 